\title{
Implementing nurse telephone follow-up clinics for monitoring parenteral osteoporosis treatment - a service evaluation
}

\author{
Authors: Sethu Mani-Babu and Rita Abdulkader
}

\section{Background}

Parenteral osteoporosis treatments including yearly intravenous zoledronate infusions and 6-monthly denosumab subcutaneous injections are increasingly used in patients unable to tolerate oral bisphosphonates. ${ }^{1}$

These treatments are generally delivered and monitored in a secondary care setting. In Ipswich Hospital this occurs mainly through the rheumatology department. Traditionally, patients receiving these treatments were followed up in the outpatient clinic by a consultant. This meant that the patient had to attend two hospital appointments a year for zoledronate treatment and four for the monitoring and administration of denosumab.

\section{Aims}

The aim of the quality improvement project, which started in 2016 as a pilot, was to improve the efficiency of the service by reducing reliance on consultant face-to-face follow-up appointments and the impact of unnecessary hospital visits on patients while continuing to deliver a high-quality and safe service. We present an evaluation of the service and its impact.

\section{Methods}

In 2016, we implemented the new pathway which still requires a first consultant rheumatologist appointment to assess the suitability of parenteral treatment. But rather than organising consultant follow up, the patient is followed up in a nurse practitioner telephone clinic supported by the consultant in charge of care. The nurse works through a safety checklist and provides advice and support to the patient. If all checks are satisfactory the patient only re-attends to receive the treatment in the hospital day unit, and for a repeat bone density scan at the end of treatment course, if applicable.

Using the rheumatology day unit database, we obtained the number of consultant follow-up appointments saved by implementing this service and calculated cost savings.

\section{Results}

Table 1 shows the number of face-to-face consultant follow-up appointments saved by implementing the new pathway.

> These changes have led to cost savings to the trust of over $£ 10,000$.

Authors: Ipswich Hospital, East Suffolk and North Essex NHS Foundation Trust

$\begin{aligned} & \text { Table 1. Face-to-face consultant follow-up } \\ & \text { appointments saved by implementing the new } \\ & \text { pathway. }\end{aligned}$
$\begin{array}{ll}\text { Near } & \text { appointments } \\ & 157 \\ 2016 & 186 \\ 2017 & 107 \\ 2018 \text { (up till August) } & \end{array}$

> The new pathway reduced the need for patients to attend the clinic saving them a journey to the hospital (especially when considering the county's size and rural setting), reduced stress related to parking and possible delays in the clinic.

> There have been no safety issues during this period, thanks to the strict adherence to the checklist and the provision of consultant support. In fact, utilising this pathway reduced the risk of delay in the provision of treatment when there are long consultant follow-up waiting lists.

> Feedback from patients and carers has been very positive.

\section{Conclusion}

We have presented the Ipswich Hospital experience in implementing nurse telephone clinics to improve the efficiency of the parenteral osteoporosis pathway. The benefits of the pathway include a reduction in hospital attendances for outpatient appointments, reducing consultant follow-up waiting lists and reducing the impact of multiple hospital visits on patients (who are commonly of older age and may have multiple comorbidities) while continuing to deliver a safe and high-quality service.

Other possible benefits include the environmental impact of the fewer numbers of car journeys, reduced pressure on hospital car parking and the impact on carers.

Maintaining the success of this pathway requires continuous investment to support the nursing and administration team and the provision of consultant support.

\section{Reference}

1 National Institute for Health and Care Excellence. Bisphosphonates for treating osteoporosis. Technology appraisal guidance [TA464]. London: NICE, 2017. www.nice.org.uk/guidance/ta464/resources/ bisphosphonates-for-treating-osteoporosis-pdf-82604905556677 [Accessed: 18 November 2018]. 